\title{
The LHCb RICH detectors
}

\author{
U. Kerzel \\ Cavendish Laboratory, University of Cambridge, JJ Thomson Avenue, Cambridge CB3 OHE, UK
}

For the LHCb RICH Collaboration

\section{A R T I C L E I N F O}

Keywords:

$\mathrm{LHCb}$

$\mathrm{RICH}$

Cherenkov

\begin{abstract}
A B S T R A C T
The LHCb experiment at the Large Hadron Collider has been optimised for high precision measurements of the charm and beauty quark sector. The different particle species produced in the high-energy collision are identified using two Ring-Imaging Cherenkov detectors.
\end{abstract}

(c) 2009 Elsevier B.V. All rights reserved.

\section{Introduction}

The main objective of the LHCb [1] experiment is to precisely determine and over-constrain the parameters of the CKM matrix, and to search for further sources of $\mathrm{CP}$ violation and new physics beyond the Standard Model in rare B-decays. Efficient particle identification at high purities over a wide momentum range from around 1 to $\approx 100 \mathrm{GeV} / \mathrm{c}$ is vital to many LHCb analyses. Fig. 1 illustrates the essential contribution of the particle identification for the case of charmless B-meson decays. Using the particle identification information the signal mode $\mathrm{B}_{s}^{0} \rightarrow \mathrm{K}^{+} \mathrm{K}^{-}$can be cleanly isolated against other decay modes.

\section{RICH detectors}

Central to the LHCb particle identification strategy are two Rich Imaging Cherenkov (RICH) detectors [2]. A schematic overview over the RICH detectors of the LHCb spectrometer is shown in Figs. 2 and 3.

In order to limit the degradation of the resolution of the tracking systems due to interaction with the detector material, the RICH photodetectors are placed outside the acceptance of the LHCb spectrometer. Using a set of spherical, coupled with flat mirrors [4,5], the Cherenkov light is projected onto the detector plane. The spherical mirrors are positioned inside the spectrometer acceptance and it is therefore of vital importance to minimise the fraction of radiation length $X_{0}$ while at the same time safeguarding the mechanical integrity of the optics. In the case of RICH 1, industrial carbon fibre has been used for the support structure and the resulting contribution to the material budget is below $6 \mathrm{~kg} / \mathrm{m}^{2}$ which corresponds to $1.5 \% X_{0}$.

E-mail address: Ulrich.Kerzel@cern.ch
Three different radiator materials are used in the two subdetectors covering a large range of momentum. In RICH 1, silica aerogel [6] is used to detect particles in the momentum range up to $\approx 10 \mathrm{GeV} / c$ and $\mathrm{C}_{4} \mathrm{~F}_{10}$ is used for $10 \leq p \leq 40 \mathrm{GeV} / c$. $\mathrm{CF}_{4}$ is used as radiator material in $\mathrm{RICH} 2$ and covers the range $16 \leq p \leq 100 \mathrm{GeV} / c$. Fig. 4 illustrates the detection thresholds of the various particle species in each of the three Cherenkov radiators.

\section{Photodetectors}

The need for a fast and efficient photo-detector operating over a large active surface area lead to the development of the custombuilt pixel Hybrid Photon Detectors (HPDs) [7] to detect the Cherenkov light produced in the radiators. The HPDs are designed and built in collaboration with industry ${ }^{1}$ Each HPD detects the converted photoelectrons using a silicon pixel-chip with 8192 active channels. Eight channels are actively OR-ed by the readout electronics to form 1024 square super-pixels which results in an effective granularity of $2.5 \times 2.5 \mathrm{~mm}^{2}$ at the entrance window. In total, $0.5 \mathrm{M}$ pixels are used to detect the Cherenkov light in an active area of $\approx 3 \mathrm{~m}^{2}$ which corresponds to $\approx 64 \%$ of the detection plane. The photodetectors are sensitive to single photons in the wavelength range from 200 to $600 \mathrm{~nm}$. The quantum-efficiency is one of the main parameters characterising the quality of the HPD. All HPDs produced exceed the quantum-efficiency of $23 \%$ at a wavelength of $270 \mathrm{~nm}$ required by the specifications. Due to significant quality progress at the manufacturer, an average quantum-efficiency of $\approx 31 \%$ was achieved.

\footnotetext{
${ }^{1}$ Photonis-DEP, BV, NL-9300 AB Roden, Netherlands as main industrial partner.
} 

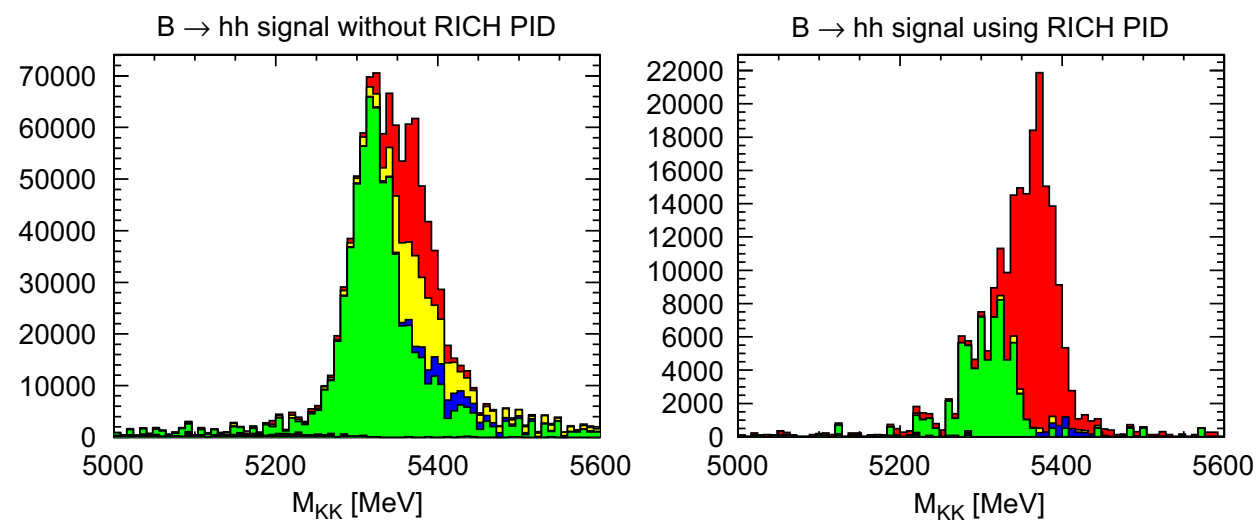

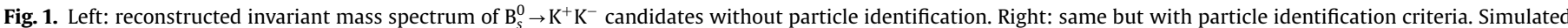
signal events are used in both figures.

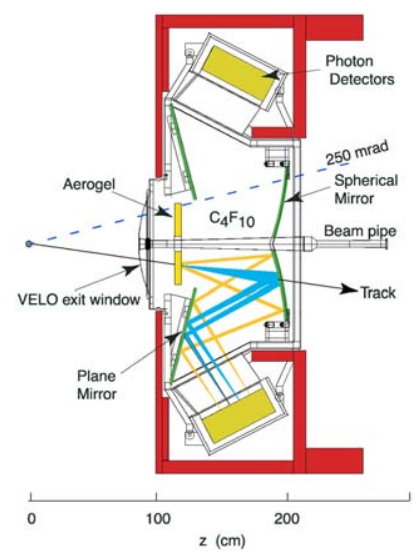

Fig. 2. Schematic overview over the RICH 1 detector of the LHCb spectrometer.

\section{Estimated performance}

Using information from the tracking system, a set of masshypotheses is assigned to each reconstructed track. This is then used to derive the probability distribution for finding photons in each pixel of the detector. A likelihood is calculated comparing the observed and anticipated hit distributions. By varying the mass hypotheses, the likelihood is maximised. Efficient $\pi$-K separation is achieved at high purity as illustrated by Fig. 5 which includes effects from all known backgrounds.

\section{Detector commissioning}

Both RICH detectors are installed in the experimental hall for more than two years and fully operational. In absence of beam, a laser and data projector are used to illuminate the photodetector plane. These are used to exercise the readout of the whole detector and for precise measurements of the HPD status and response. In addition, alignment studies of the individual HPDs can be performed using known patterns and the subsequent analysis of the measured distribution. Furthermore, data taken when the $\mathrm{LHCb}$ magnet was switched on allows to study the effect of magnetic distortions of the patterns projected onto the photodetector plane.

At the start of the LHC commissioning with beam in September 2008 bunches of protons were transferred from the SPS into the injection line of the LHC and then stopped by a beam stopper several hundred metres ahead of LHCb. The resulting secondary particles traversed the LHCb spectrometer. Fig. 6 shows the

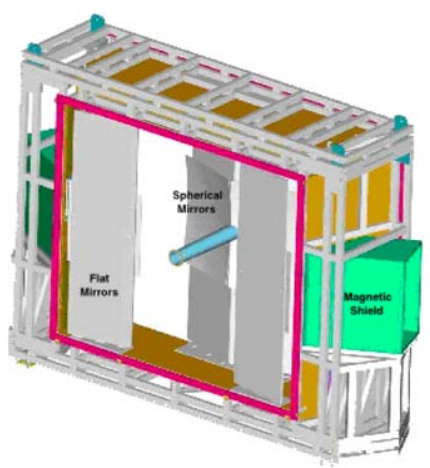

Fig. 3. Schematic overview over the RICH 2 detector of the $\mathrm{LHCb}$ spectrometer.

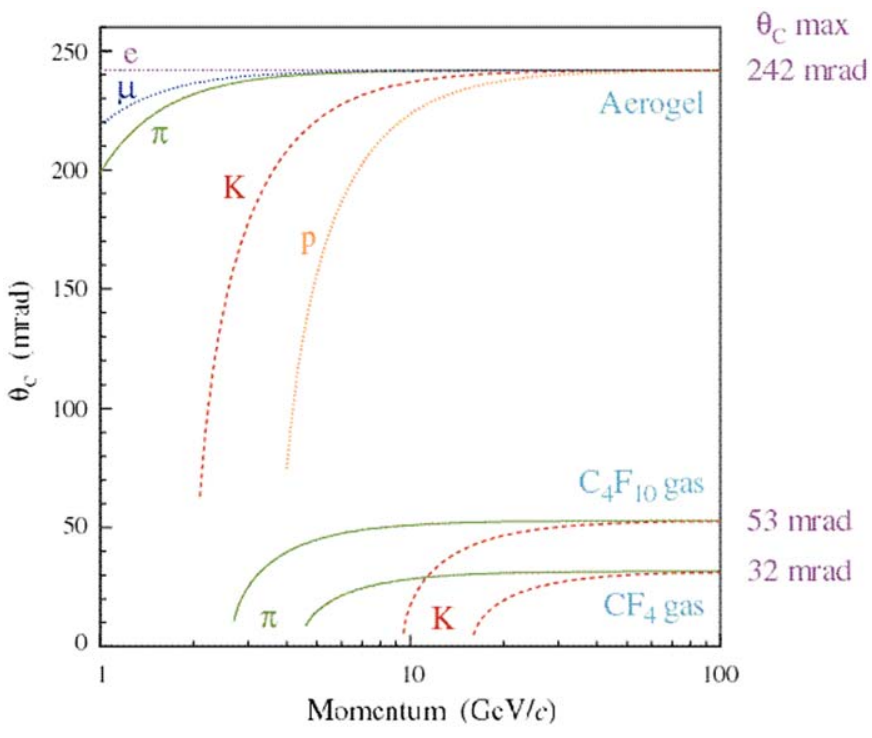

Fig. 4. Detection thresholds for the different particle species in the three Cherenkov media.

response of the RICH 2 detector to such an event. Due to the high number of secondary particles produced in the beam stopper, most photodetectors effectively saturate and each pixel of the silicon pixel chip is activated. 


\section{Beam test}

Using the SPS facility at CERN, a set of 48 HPDs were tested in 2006 using a beam matching the 25 ns structure of the LHC. HPDs and DAQ electronics were taken from the final production and placed in a special test setup which was already used in earlier tests [8]. The events have been recorded using an early version of the LHCb online software environment. The subsequent analysis has been performed using the official LHCb reconstruction and analysis

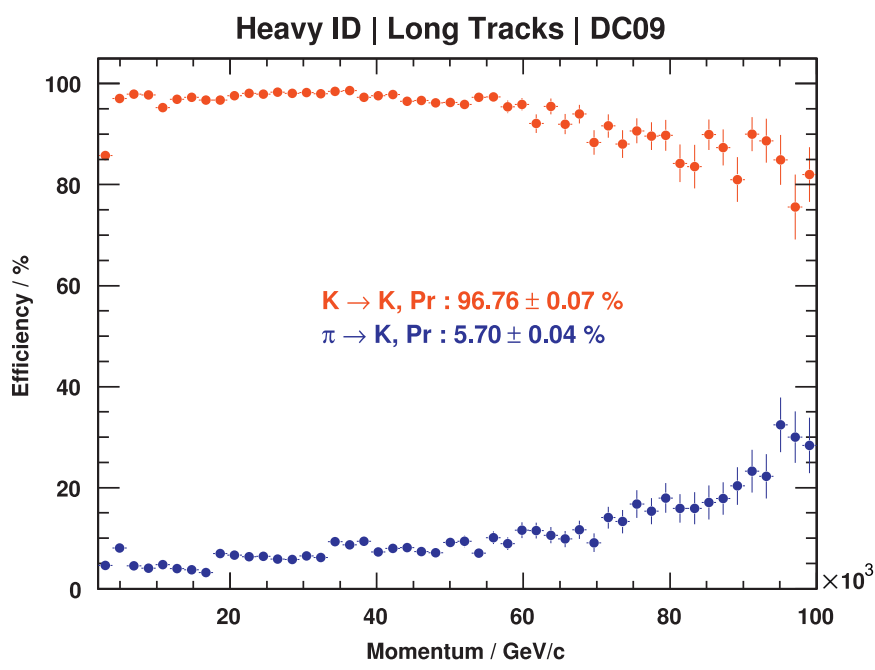

Fig. 5. Estimated performance of the particle identification based on simulation studies. software; simulated events have been obtained using the full $\mathrm{LHCb}$ simulation package where the detector geometry description has been modified to represent the setup used in the beam test. It has been successfully demonstrated [9] that all aspects of the RICH operation and data analysis from detection of the photons to the

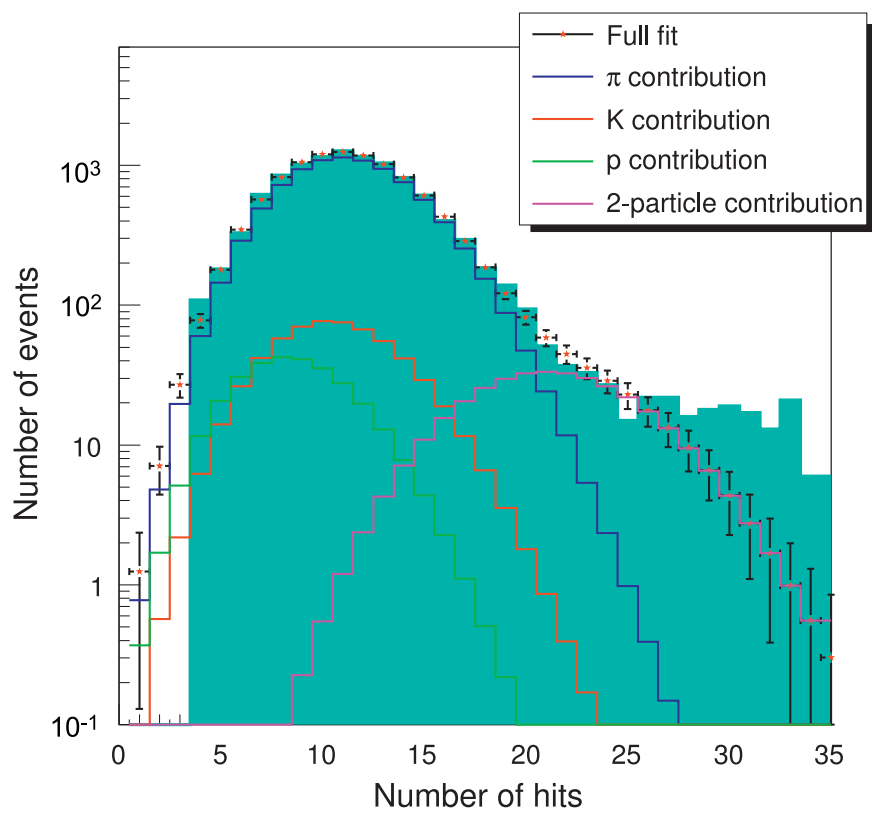

Fig. 7. Fit to the photon yield including contributions from $\pi, K, p$ and multiple particles.

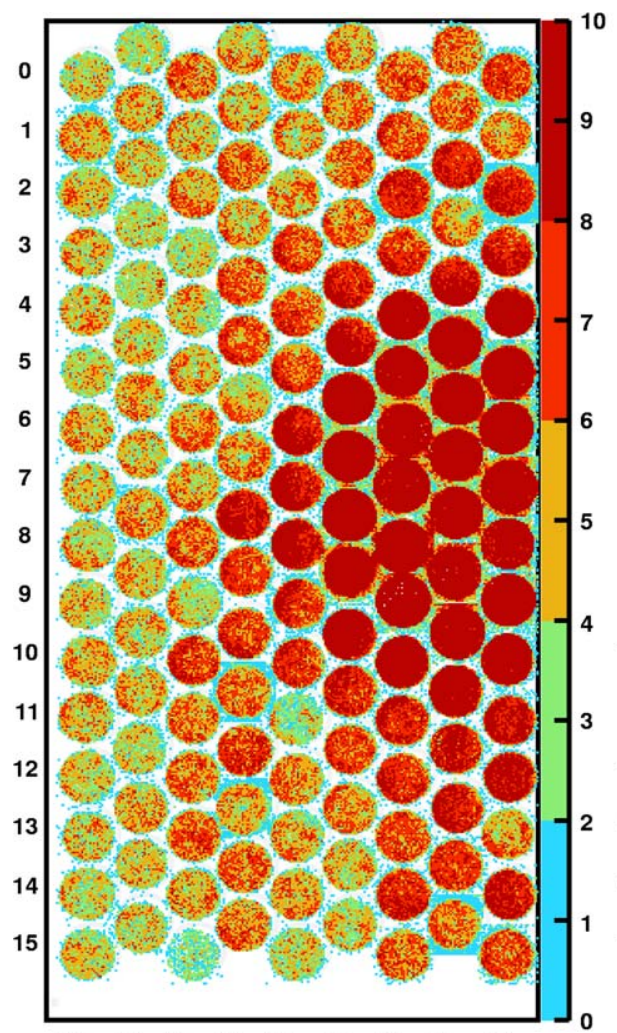

$\begin{array}{llllllllll}\text { A8 } & \text { A7 } & \text { A6 } & \text { A5 } & \text { A4 } & \text { A3 } & \text { A2 } & \text { A1 } & \text { A0 }\end{array}$

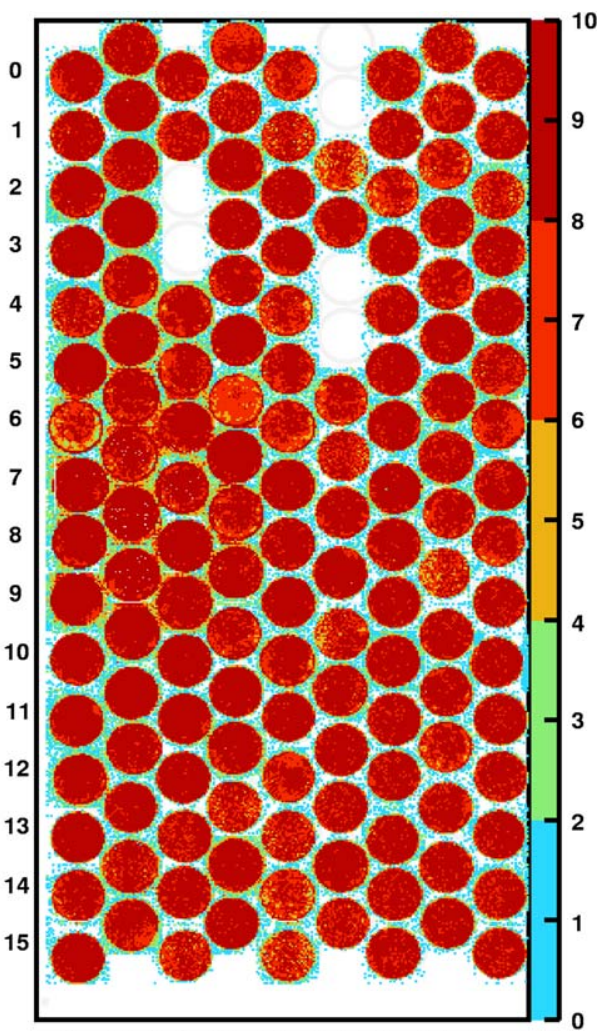

$\begin{array}{llllllllll}\mathrm{C} 8 & \mathrm{C} 7 & \mathrm{C} 6 & \mathrm{C} 5 & \mathrm{C} 4 & \mathrm{C} 3 & \mathrm{C} 2 & \mathrm{C} 1 & \mathrm{C} 0\end{array}$

[Looking at front face of the photocathodes]

Fig. 6. Detector response during LHC tests. 


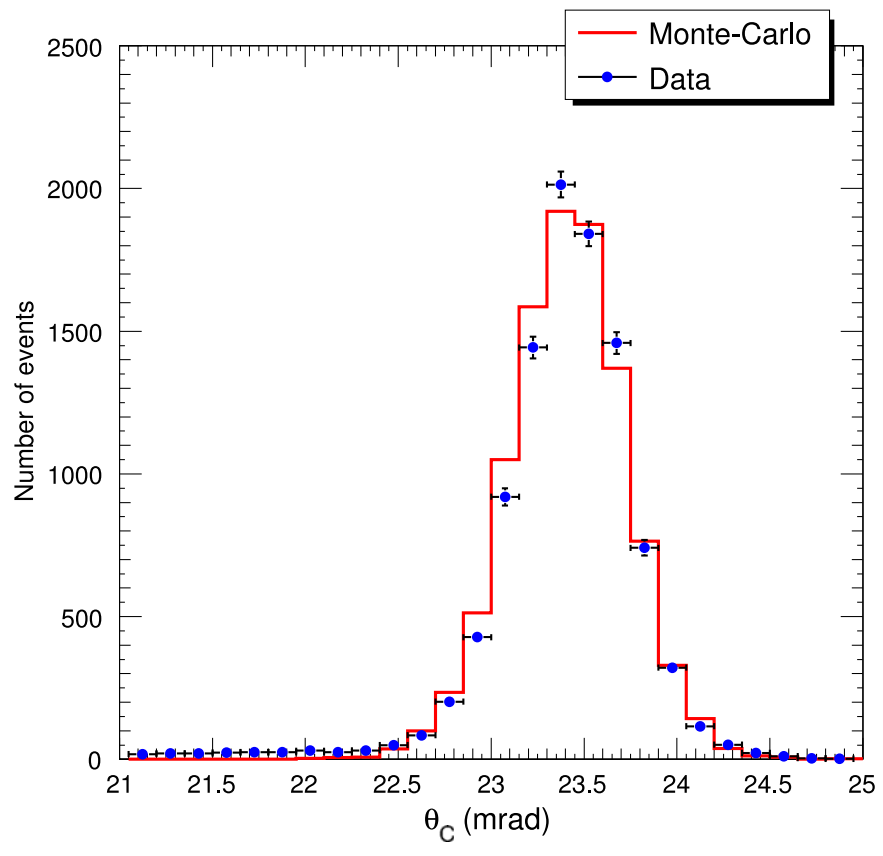

Fig. 8. Comparison of the Cherenkov angle resolution measured in data and realistic simulation. The resolution is measured as $\sigma_{\theta}=(0.296+0.003$ (stat.) \pm 0.006 (syst.)) mrad. $\mathrm{N}_{2}$ has been used as radiator material in both cases.

analysis of the recorded data meet the stringent requirements of the LHCb experiment. Fig. 7 shows a fit to the measured photon yield from the HPDs. The fit accurately reproduces the spectrum according to the various particles present in the beam. Fig. 8 shows the Cherenkov angle resolution $\sigma_{\theta_{c}}$ measured in the data. The Cherenkov angle resolutions are measured to be $\sigma_{\theta}=(0.296 \pm 0.003$ (stat.) \pm 0.006 (syst.)) $\mathrm{mrad}$ and $\sigma_{\theta}=(0.166 \pm 0.002$ (stat.) \pm 0.002 (syst.)) mrad for $\mathrm{N}_{2}$ and $\mathrm{C}_{4} \mathrm{~F}_{10}$, respectively. These values are in line with expectations and both the measured resolution and the photon yield confirm the results of previous beam-tests [8].

\section{Conclusion and current status}

The LHCb RICH project is ready for data-taking after the start of the LHC. Based on beam tests and commissioning of the detector the performance of the RICH detectors is expected to meet the stringent requirements of the $\mathrm{LHCb}$ physics programme.

\section{References}

[1] The LHCb Collaboration, JINST 3 (2008) S08005.

[2] LHCb RICH Technical Design Report CERN/LHCC/2000-037, LHCb TDR 3, September 2000.

[4] G. Barber, et al., Glass-coated beryllium mirrors for the LHCb RICH1 detector, LHCb-2006-007, 2006.

[5] F. Metlica, Development of light-weight spherical mirrors for RICH detectors, in: Contribution to Sixth International Workshop on Ring Imaging Cherenkov Counters, 2007.

[6] T. Bellunato, et al., Nucl. Phys. Proc. Suppl. 150 (2006) 281.

[7] M. Moritz, et al., IEEE Trans. Nucl. Sci. NS-51 (3) (2004) 1060-1066.

[8] M. Adinolfi, et al., Nucl. Instr. and Meth. A 574 (2007) 39.

[9] M. Adinolfi, et al., Nucl. Instr. and Meth. A 39651 (2009). 